\title{
A Case of Fetal Cardiac Rhabdomyoma
}

\author{
Chitra Ganesh, Anjana Sridhar
}

\section{ABSTRACT}

Rhabdomyoma is one of the commonest cardiac tumor detected in prenatal ultrasound. Though its benign and self-limiting, it has a strong association with tuberous sclerosis, and hence needs thorough evaluation not only of the fetus but of the parents for signs of tuberous sclerosis. When isolated, fetus with rhabdomyoma needs to be only followed up for any cardiac complications associated with the mass and delivered.

Keywords: Rhabdomyoma, Tuberous sclerosis, Autosomal dominant, Echocardiography, Counseling.

How to cite this article: Ganesh C, Sridhar A. A Case of Fetal Cardiac Rhabdomyoma. Int J Infertility Fetal Med 2013;4(2):66-69.

\section{Source of support: Nil}

\section{Conflict of interest: None}

\section{INTRODUCTION}

Fetal cardiac rhabdomyoma is a benign, smooth muscle tumor $^{1}$ of the myocardium consisting of embryonal myoblasts. About 60 to $90 \%$ of cardiac rhabdomyomas ${ }^{2,3}$ are associated with or have a positive family history of tuberous sclerosis which is an autosomal-dominant hamartomatous condition. The remaining cases can present as isolated conditions.

We had a case of an isolated rhabdomyoma who went on to have an uneventful antenatal and postnatal period. We present this case report and discussion about rhabdomyoma.

\section{CASE REPORT}

A 22-year-old, primigravida, of a nonconsanguineous marriage and spontaneous conception, was referred to our center at 28 to 29 weeks for second opinion, for a mass in the heart advised. The mid-trimester anomaly scan done outside was reported to be normal.

At our center, ultrasound ${ }^{4}$ examination revealed a single, live fetus, corresponding to 28 to 29 weeks of gestation, with multiple echogenic intracardiac masses, the largest in the right ventricle, measuring $1.7 \times 1.1 \mathrm{~cm}$ (Figs $1 \mathrm{~A}$ and $\mathrm{B}$ ). The rest of the cardia appeared structurally normal, as also the rest of the fetal anatomy.

Diagnosis of cardiac rhabdomyoma was made, and a follow-up scan after 4 weeks was advised. Follow-up at

Date of Received: 20-08-13

Date of Acceptance: 28-08-13

Date of Publication: May 2013
33 weeks revealed (Fig. 2) no significant change in the mass size, with no further complications.

Rest of the pregnancy was uneventful and the patient delivered a healthy female infant weighing $2.8 \mathrm{~kg}$ at her native place.

Postnatal echocardiography on days 5 revealed normal study of the heart with no evidence of cardiac masses.

Clinically, the infant appeared completely normal. Thorough clinical examination of both parents revealed no evidence of tuberous sclerosis. ${ }^{5}$ Pedigree analysis revealed no family history of tuberous sclerosis.

\section{DISCUSSION}

Fetal cardiac tumors constitute 1 to 2 per 10,000 births. Around $90 \%$ of these are benign, out of which 60 to $80 \%$ are rhabdomyomas, others being fibromas, teratomas, vascular tumors and very rarely myxomas.
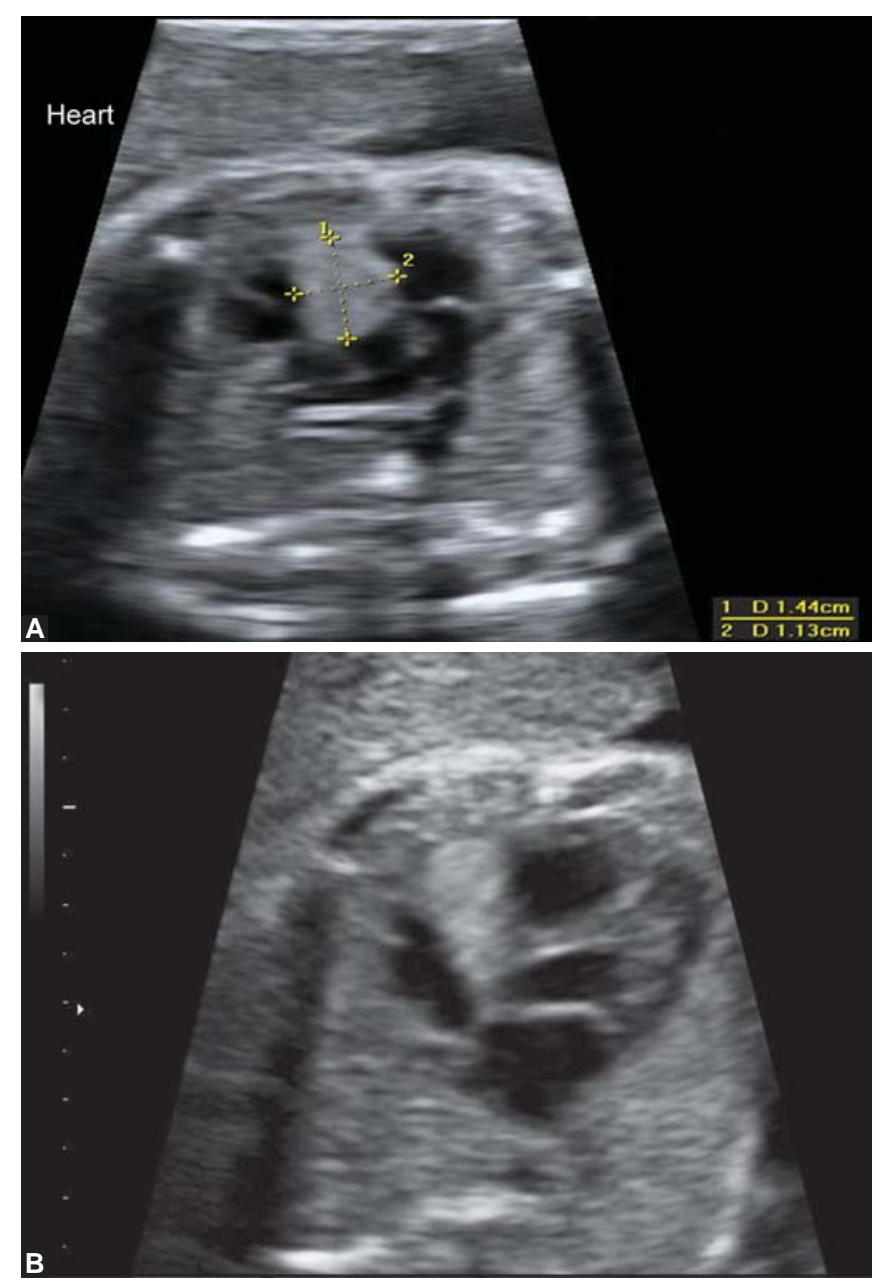

Figs 1A and B: Homogenous echogenic mass in the wall of the right ventricle 
Rhabdomyomas are the most common primary tumors of the heart, occurring in fetus, neonates and mostly identified in the first year of life, accounting for $1 \%$ of all cardiac disorders. These are the hamartomas of the developing myocytes. These tumors vary in size and number. They are usually multiple, commonly arising from the ventricular myocardium of either side of the heart. In 30\% of the cases, they arise from the atria and rarely also from endocardium, pericardium, papillary muscles, valves or outflow tracts. They may have a mild atypical histological presentation without invasion or metastasis. Though benign, positioning in the critical areas can lead to lethal arrhythmias and chamber obstruction. The natural history is that of a spontaneous regression.

The exact etiology is not known. It can occur as isolated cases in sporadic mutation. Usually associated with genetic disorder-tuberous sclerosis in 60 to $90 \%$ of the cases, they are also associated rarely with major cardiac anomalies, like TOF and Ebstein's anomaly.

Tuberous sclerosis is an autosomal dominant neurocutaneous disorder, resulting from hamartomatous growth that can occur in any organ- brain, kidneys, heart, skin, eyes and others. There is an age-related presentation of lesions in different organs. About $50 \%$ of the patients develop rhabdomyoma as a fetal manifestation. The classic presentation (Figs 3A and B) of tuberous sclerosis is in later life with hallmark findings of facial angiofibromas, seizures and mental retardation. Diverse cutaneous manifestations like café-au-lait patches (light brown areas), hypochromatic patches, Shagreen patches are also seen.

Renal cysts, multiple and bilateral angiofibroma of the kidney, brain manifestations like sub-ependymal nodules, subcortical tubers, ependymomas of the third ventricle and astrocytomas may also be present. Greater the number of tubers, greater is the neurologic impairment. They can cause obstruction and hydrocephalus when they extend into the ventricles in the foramen of Monro area. Early manifestation of seizures leads to more severe mental retardation. Magnetic resorance imaging (MRI) is more helpful in the diagnosis of brain lesions.

Histologically, rhabdomyomas present as anomalous benign proliferation of embryonal myoblasts, displaying typical spider cells (Fig. 4).

The diagnosis is usually by two-dimensional Doppler echocardiography which also helps to monitor their influence on the fetal cardiac function. They present as single or multiple, round, usually well-delineated homogeneous (echogenic) masses resembling small uterine fibroids. Around 60 to $90 \%$ of children diagnosed with cardiac rhabdomyomas demonstrate clinical or radiologic evidence of tuberous sclerosis or have a positive family history. They are seen after 20 to 22 weeks, during an anomaly scan (infact, finding of rhabdomyoma during routine second trimester scan may lead to the recognition that the mother is affected), maximal growth occurs between 22 and 32 weeks and, rarely, grows after 35 weeks, and can remain asymptomatic. Usually, they do not regress during pregnancy (as maternal hormones are implicated), stop

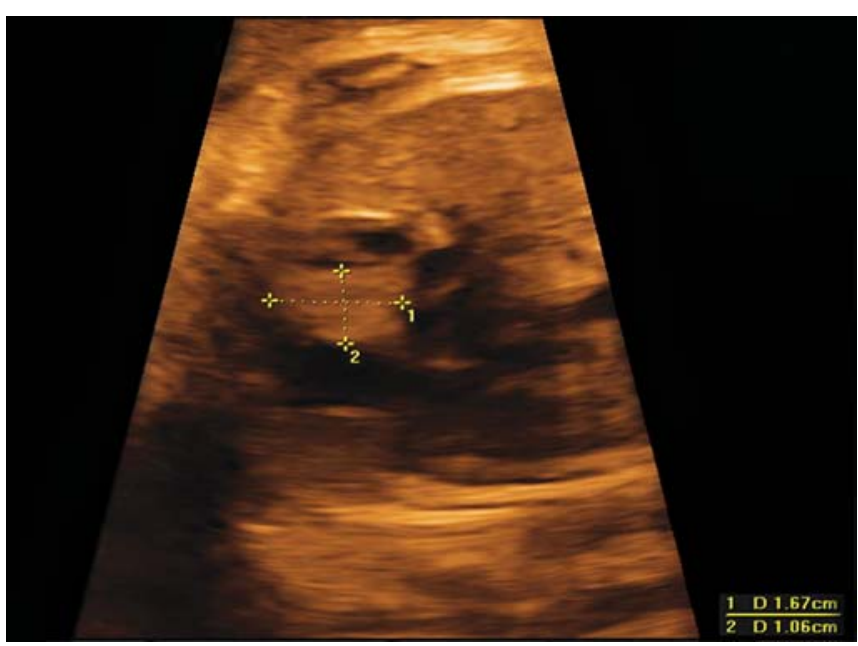

Fig. 2: No significant change in the mass size
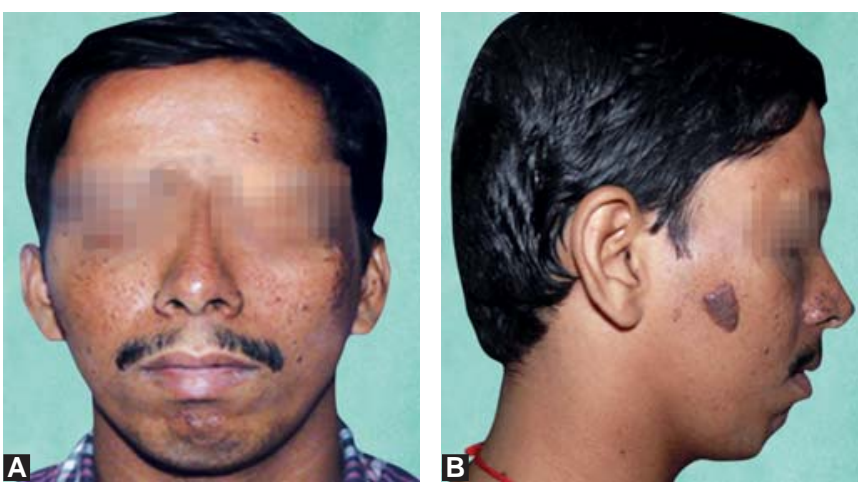

Figs 3A and B: Facial angiofibromas

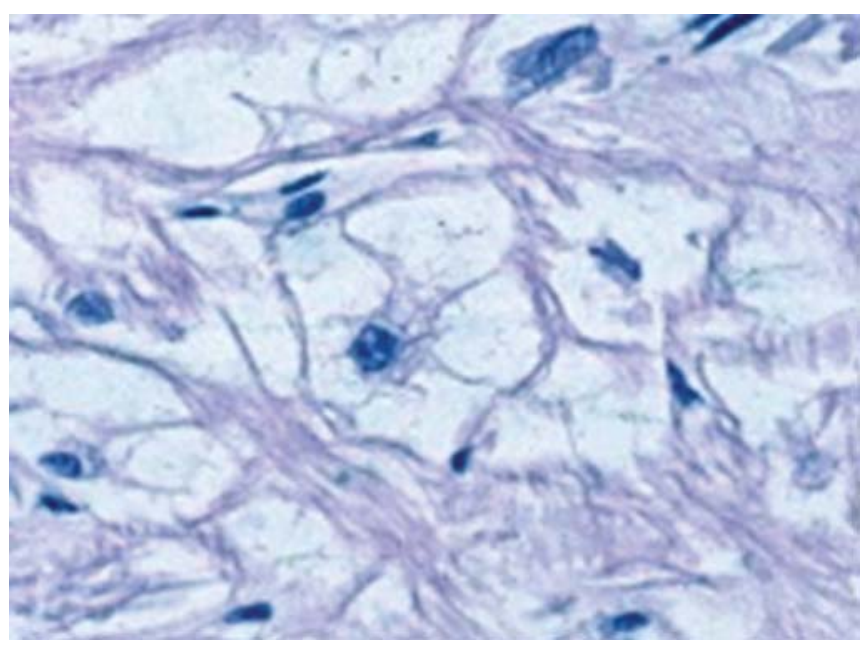

Fig. 4: Spider cells 
growing postnatally and later regress. Location of the tumor in the critical areas can lead to complications as follows:

Disruption of the intracardiac blood flow-out flow tract obstruction, valvular insufficiency, myocardial dysfunction, CCF and hydrops.

Interference with conducting system — arrhythmias, both atrial and ventricular, either due to over growth of Purkinje cells with increased impulse conduction, or, disruption of conducting tissue by tumor growth within the IVS (SVT, WPW syndrome, atrial flutter/fibrillation, AV block, ectopic atrial tachycardia, ventricular tachycardia).

Death-can be due to disruption of the blood flow, arrhythmias, valve stenosis or loss of functional myocardium.

\section{Differential Diagnosis}

Multiple tumors are always rhabdomyomas unless proved otherwise.

If single,

1. Fibroma - the second most common tumor presenting as an echogenic mass (Fig. 5), arising from ventricular septum or myocardium, bulging into the cavity,

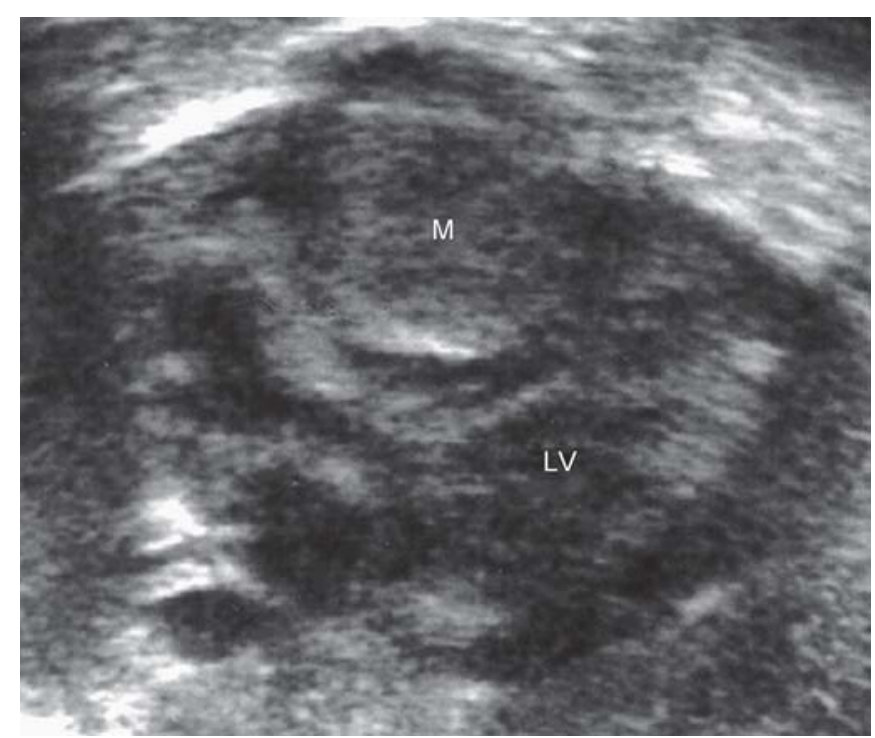

Fig. 5: Fibroma
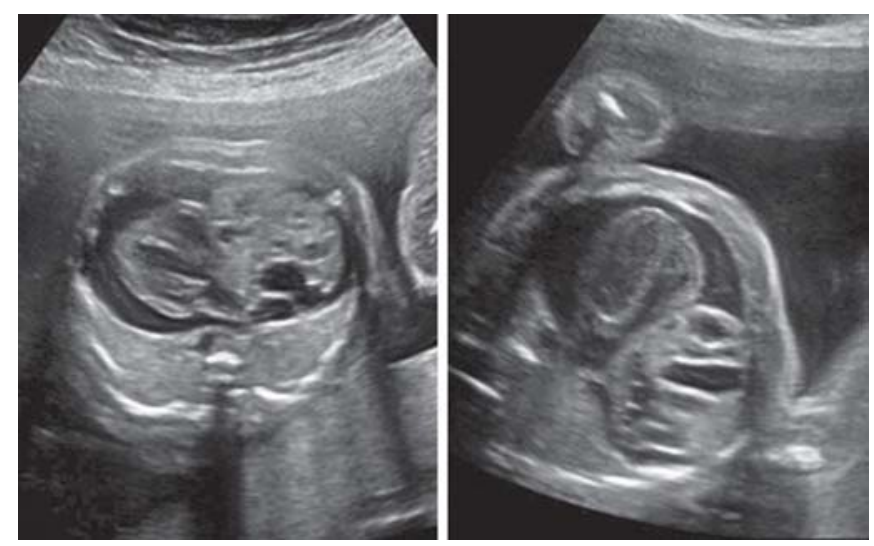

Fig. 6: Teratoma with pericardial effusion resembling a uterine myoma. Calcifications are typical to differentiate from rhabdomyoma.

2. Teratoma - complex mass (solid and cystic area), located in the pericardial cavity causing pericardial effusion. Derivatives of all three germ layers can be seen (Fig. 6).

3. Cardiac hemangioma-vascular tumors can arise from any chamber, usually from right atrium, can present as complex echogenic mass with solid and cystic areas with calcification. It can be intramural or endocardial.

4. Myxoma-endocardium-based lesion without infiltration, usually seen in the left atrial side of the interatrial septum, appears moderately echogenic. Myxomas are very rare during fetal period.

Extracardiac lesions like CCAM and BPS can mimic rhabdomyoma but careful echo can differentiate.

\section{Management of Pregnancy}

There are two main considerations in the management as follows:

1. Evaluation of the cardiac function with detailed echo: Standard management of pregnancy is appropriate for uncomplicated cases, delivery at tertiary center in the presence of a pediatric cardiologist.

2. Evaluation for other signs and symptoms of tuberous sclerosis: A genetic counseling with the help of medical geneticist-detailed three-generation pedigree, thorough examination of the parents and siblings for signs and symptoms of tuberous sclerosis (major and minor criteria).

A detailed scan for evidence of renal cysts or cerebral abnormalities.

Fetal MRI for presence of subependymal nodules.

a. A fetus with rhabdomyoma and renal cysts or subependymal nodules, independent of family history has presumptive clinical diagnosis of tuberous sclerosis.

b. In the presence of a positive family history, fetus with rhabdomyoma is presumed to be affected. Question of termination can be discussed. Fetal karyotyping is not indicated, but DNA analysis of the fetal cells can be done (amniocentesis or umbilical cord blood ) to identify in TSC1 or TSC2. Fetal intervention for rhabdomyoma is nil at present.

\section{Prognosis}

Good for isolated uncomplicated rhabdomyoma. Otherwise, it depends on presence of other complications. Due to the great variability of expression of tuberous sclerosis, accurate prediction of status of the child is difficult from the parent status. Mental prognosis varies with the locus of defective gene which may influence the decision about future pregnancy. 


\section{Management of the Newborn ${ }^{7}$}

- Delivery at tertiary center in the presence of a pediatric cardiologist, postnatal physical examination, X-ray, ECG, four extremity BP measurement, postnatal echo, renal sonogram and MRI, medical genetic consultation.

- Conservative management in asymptomatic neonate.

- Correction of arrhythmias in affected neonates.

- Surgery only with hemodynamic compromise.

- Newborn with cortical tubers will be at risk for seizures and infantile spasms and may need anticonvulsant therapy.

- Children with tuberous sclerosis to be followed-up with MRI and renal ultrasound every 1 to 3 years.

\section{Long-term Outcome}

Variable, if due to tuberous sclerosis, from normal to severe mental retardation. About $38 \%$ of TS will have developmental delays. Those, who develop seizures in the first year, will have more severe mental disabilities.

Later, they can develop renal and pulmonary angiolipomas, multiple skin findings which will have a cosmetic issue.

When there is a family history, the occurrence in future generations varies as well. This means a parent of normal intelligence, who is diagnosed with TS based on the findings of fetal ultrasound, can have a child who is severly affected.

\section{Counseling/Genetics}

Isolated rhabdomyoma, sporadic, low recurrence rate but needs close monitoring.

Tuberous sclerosis (C) defects and mutations are on two tumor suppressor genes.

TS C1 gene on chromosome 9 and codes for protein hamartin.

TS C2 gene on chromosome 16 and codes for protein tuberin (more severe phenotype).

These proteins act as tumor suppressors, agents that regulate cell proliferation and differentiation.

Penetrance for known TS is $100 \%$ but the phenotypic expression varies.

Recurrent risk is low in new mutation (sporadic), medium in somatic mutation and high in gonadal mosaicism. It is $50 \%$ in parental affection.

It is suspected that $10 \%$ may have gonadal mosaicism. This means that the parent has a mutation of a gene in one of the sex linked chromosomes ( $\mathrm{X}$ or $\mathrm{Y}$ ). The parent will present without TS and when genetic testing is performed, the typical genes associated with TS will not be evident. Only the sex linked cells are involved and only some of them will have the mutation while others will be normal. For this reason, some families have recurrent cases of children affected with TS even though neither parent is affected.

\section{ACKNOWLEDGMENT}

We would like to thank Dr Shobha Rani, MD, Kumar Nursing Home, Channarayapatna, Department of Genetic Counseling, MediScan, Chennai, Tamil Nadu, India, for her valuable support.

\section{REFERENCES}

1. Isaacs H Jr. Fetal and neonatal cardiac tumors. Pediatr Cardiol 2004;25(3):252-273. Pmid: 15360117.

2. Bader RS, Chitayat D, Kelly E, Ryan G, Smallhorn JF, Toi A, Hornberger LK. Fetal rhabdomyoma: prenatal diagnosis, clinical outcome, and incidence of associated tuberous sclerosis complex. J Pediatr 2003;143(5):620-624.

3. Chao AS, Chao A, Wang TH, Chang YC, Chang YL, Hsieh CC, Lien R, et al. Outcome of antenatally diagnosed cardiac rhabdomyoma: case series and a meta-analysis. Ultrasound Obstet Gynecol 2008;31(3):289-295.

4. Callen PW. Ultrasonography in obstetrics and gynecology. 5th edition. Dec 12, 2007.

5. Gamzu R, Achoron R, Hegesh J, et al. Evaluating the risk of tuberous sclerosis in cases with prenatal diagnosis of cardiac rhabdomyoma. Prenat Diagn 2002;22:1044.

6. D’Addario V, Di Naro E, et al. Prenatal diagnosis and postnatal outcome of cardiac rhabdomyomas. J Perinat Med 2002; 30:170.

7. Bianchi DW, Crozleholme TM, D'Alton ME, Malone FD. Fetology-diagnosis and management of fetal patient. 403-408.

\section{ABOUT THE AUTHORS}

\section{Chitra Ganesh (Corresponding Author)}

Consultant, Department of Fetal Medicine, Karthik Ultrasound Scan Centre and Fortis Hospital, Bengaluru, Karnataka, India, e-mail: chitra.ganesh@yahoo.co.in, website: www.karthikultrasound.com

\section{Anjana Sridhar}

Consultant, Department of Obstetrics, Gynecology and Sonology Karthik Ultrasound Scan Centre, Bengaluru, Karnataka, India 